\title{
Vegetation shifts and microbial substrate utilisation in boreal forest floors of Western Canada.
}

\author{
EMILY LLORET ${ }^{1}$ AND SYLVIE QUIDEAU $^{2}$ \\ ${ }^{1}$ Université de Lille / LGCgE \\ ${ }^{2}$ University of Alberta / Faculty of Agricultural, Life and \\ Environmental Sciences \\ Presenting Author: emily.lloret@univ-lille.fr
}

Boreal forest soils are highly susceptible to global warming, and in the next few decades, are expected to face large increases in temperature and transformative vegetation shifts. The entire boreal biome will migrate northward, and within the main boreal forest of Western Canada, deciduous trees will replace conifers. The main objective of our research is to assess how these vegetation shifts will affect functioning of soil microbial communities and ultimately the overall persistence of boreal soil carbon. In this study, aspen and spruce forest floors from the boreal mixedwood forest of Alberta were incubated in the laboratory for 67 days without (control) and with the addition of three distinct ${ }^{13} \mathrm{C}$ labelled substrates (glucose, aspen leaves and aspen roots). Specifically, addition of aspen litter to spruce forest floor mimicked future vegetation shifts. Tracking of carbon utilization by microbial communities in both aspen and spruce forest floors was accomplished using ${ }^{13} \mathrm{C}$ - PLFA analysis, and ${ }^{13} \mathrm{C}-\mathrm{CO}_{2}$ measurements allowed quantification of the relative contribution of each added substrate to microbial respiration. Microbial communities from both forest floors rapidly utilized and respired the added glucose, but the aspen community was the only one showing a positive priming effect four hours following addition. The aspen community was also more efficient at recycling the added glucose and showed a greater ${ }^{13} \mathrm{C}$-PLFA enrichment than the spruce community even at the end of the 67day incubation. Following addition of aspen leaves and roots, respired $\delta^{13} \mathrm{CO}_{2}$ was more positive for the aspen than the spruce community, indicating that the added litter contributed relatively more to total respiration. On the other hand, a greater ${ }^{13} \mathrm{C}$-PLFA enrichment was observed for the spruce community. These results suggest that in the case of a shift from spruce to aspen, relatively more of the aspen litter would be retained in soils by the spruce microbial community. 